\title{
Las ciudades y el sol: Paridad de red de la generación de electricidad con sistemas fotovoltaicos en los hogares en Colombia
}

\author{
Lorena Cadavid ${ }^{1} \mid$ Kathleen Salazar Serna ${ }^{2}$ | Alejandro Valencia Arias ${ }^{3}$ | Carlos Jaime Franco ${ }^{4}$ \\ Recibido: 23-07-2019 | en su versión final: 19-02-2020
}

Resumen

\begin{abstract}
La autogeneración de electricidad con fuentes solares, se ha dinamizado a nivel mundial en varias regiones, las cuales han implementado esquemas de ventas de electricidad de los hogares a la red. Este artículo, evalúa el ahorro que representa para los hogares, la implementación de estos esquemas con autogeneración de electricidad a partir de fuentes solares, en territorios con costos de electricidad moderados y alta radiación solar. Lo cual, amplía el entendimiento de las nuevas tecnologías de autogeneración, para los hogares en territorios de América Latina. Asimismo, cuantifica el impacto de las reglamentaciones de ventas de electricidad en los territorios, empleadas en la masificación de los sistemas fotovoltaicos. Se desarrolló un modelo financiero que proyecta los flujos de caja de la implementación de un sistema fotovoltaico en un hogar promedio, y los compara con los flujos de caja al satisfacer la demanda a través de las compras de electricidad a la red nacional. Los resultados se analizan con tres indicadores financieros: costo nivelado de la electricidad, tasa interna de retorno y período de recuperación de inversión. Adicionalmente, se calcula la disminución de emisiones de dióxido de carbono, al reemplazar la electricidad generada con la fuente solar. La implementación de esquemas de ventas a la red, incrementa los ahorros de los hogares con la autogeneración de electricidad con fuentes solares. Para el caso de Medellín, la nueva reglamentación permitió un 20\% de ahorros adicionales y alcanzar la paridad de red para configuraciones sin baterías, con una capacidad de generación de 0,7kW.
\end{abstract}

Palabras clave: energía solar; panel solar; autogeneración a pequeña escala; análisis financiero

Citación

Cadavid, L. et al. (2020). Las ciudades y el sol: Paridad de red de la generación de
electricidad con sistemas fotovoltaicos en los hogares en Colombia. ACE:
Architecture, City and Environment, $15(43)$,
http://dx.doi.org/10.5821/ace.15.43.8772

\footnotetext{
${ }^{1}$ Profesora del Departamento de Ciencias de la Computación y la Decisión de la Facultad de Minas, Universidad Nacional de Colombia - sede Medellín. Profesora del Departamento de Ciencias Administrativas, Instituto Tecnológico Metropolitano, Medellín, Colombia (ORCID: 0000-0002-60255940), ${ }^{2}$ Profesora del Programa de Ingeniería Industrial en la Pontificia Universidad Javeriana Cali, Colombia (ORCID: 0000-0003-3824-7044), ${ }^{3}$ Profesor del Departamento de Ciencias Administrativas, Instituto Tecnológico Metropolitano, Medellín, Colombia (ORCID: 0000-0001-9434-6923, WoS Researcher ID: E-5174-2017, Scopus autor ID: $\underline{5250034100)}$, ${ }^{4}$ Profesor del Departamento de Ciencias de la Computación y la Decisión de la Facultad de Minas, Universidad Nacional de Colombia - sede Medellín, Colombia (ORCID: 0000-0002-7750-857X) Correo electrónico de contacto: kathleen.salazar@javerianacali.edu.co
} 


\title{
The Cities and the Sun: The Grid Parity of Electricity Generation with Photovoltaic Systems in Colombian Households
}

\begin{abstract}
The self-generation of electricity from solar sources has been boosted worldwide in several regions, which have implemented electricity sales policies from households to the grid. This paper aims at evaluating household savings by implementing these policies. The auto-generation of electricity from solar sources is evaluated in territories with moderate electricity costs and high solar radiation. A financial model forecasts the cash flows for a photovoltaic system in a typical household. The model compares it to the cash flows of buying the electricity from the national grid, by analyzing three financial indicators: the levelized cost of electricity, the internal rate of return of the project, and the recovery period of the investment. Furthermore, the possible scenario of carbon dioxide emission reduction is analyzed. The implementation of grid sales schemes increases the savings of households with the self-generation of electricity from solar sources. In the case of Medellín city, the new regulation for sales allowed an additional 20\% savings. Medellín achieves network parity for configurations with a generation capacity of $0,7 \mathrm{~kW}$ without batteries. This article broadens the understanding of new self-generation technologies for households in Latin American territories. Furthermore, it quantifies the impact of electricity sales regulations in the territories, used for the popularization of photovoltaic systems.
\end{abstract}

Keywords: solar energy; solar panel; small-scale self-generation; financial analysis

\section{Introducción}

\subsection{Generación solar de electricidad en los hogares}

Los sistemas fotovoltaicos para la generación de electricidad (en adelante SFV), se han reconocido internacionalmente como una tecnología con un vasto potencial (Bazilian et al., 2013; Bhandari \& Stadler, 2009; Breyer \& Gerlach, 2013). Razones como la disponibilidad del sol a nivel mundial y la generación limpia de estos sistemas, han contribuido con este fenómeno. La generación limpia, se refiere a la producción de energía eléctrica con un impacto mínimo o nulo al ecosistema, obteniendo energía eficiente, pero sostenible (NC Sustainable Energy Association [NCSEA], 2019; Zhang \& Wu, 2012). Asimismo, la energía utilizada durante la fabricación de paneles fotovoltaicos es mucho menor de lo que generarán durante su vida útil; incluso, con bajos niveles de sol como los reportados en el Reino Unido, un SFV generará la energía usada en su producción (emboided energy) en un período de 3 años, lo que le confiere un balance energético conveniente (Centre for Alternative Technology [CAT], 2019).

A pesar de los altos precios que inicialmente hicieron esta tecnología solar inviable, la rápida reducción en los costos la ha convertido en una opción económicamente factible para naciones y hogares. En efecto, los precios de los SFV se han reducido en un 74\% entre 2009 y 2018, y se espera que los precios continúen reduciéndose en los próximos años (IRENA, 2019). Como resultado de las reducciones continuas en el precio de los equipos y la implementación de políticas gubernamentales, la capacidad mundial de generación de electricidad con sistemas fotovoltaicos, ha crecido más rápido 
que con otras fuentes renovables (International Energy Agency, 2018). Esta capacidad pasó de 40 GW a aproximadamente 500 GW, entre los años 2010 y 2018 (IRENA, 2019; REN 21, 2019). Esto quiere decir que, el 2,4\% de la electricidad generada en todo el planeta en el año 2018, provino de fuentes solares, mientras que la capacidad instalada de generación solar, representa alrededor del 7,2\% a nivel mundial (REN 21, 2019). La diferencia entre el porcentaje de capacidad instalada y el porcentaje de generación, se explica por la intermitencia de la fuente solar, que hace que el factor de capacidad de las plantas solares sea inferior al de una hidroeléctrica o una planta nuclear, siendo en promedio entre 10\% y 30\% frente a 40\% y 90\%, respectivamente (U.S. Energy Information Administration [EIA], 2019). Es decir, $480 \mathrm{GW}$ (potencia) instalados en plantas solares podrían generar entre 48 GW y 144 GW en 1 hora (consumo), mientras la misma cantidad de GW instalados en hidroeléctricas, generarían entre 244 GWh y 432 GWh aproximadamente.

A nivel residencial, la paridad de red solar se ha alcanzado para varias regiones del mundo, y se espera que se alcance para otras regiones en el año 2020 (World Energy Council, 2016). Países en Europa y otros como Estados Unidos, Brasil, Australia y China, cuentan con regiones que alcanzaron la paridad de red solar desde el año 2015 (Deutsche Bank report, 2015). Esto significa, que el costo de la electricidad generada a partir de un SFV residencial, es similar o inferior al precio de la energía de la red eléctrica nacional. De esta manera, los hogares pueden realizar sus propios análisis para determinar si es conveniente o no, generar su propia electricidad a partir de un SFV, dados los costos locales y su consumo eléctrico. Los análisis que indican paridad son particularmente útiles en el diseño de políticas públicas para la difusión de la tecnología solar si se considera la variedad de motivaciones que respaldan la intención individual de tener hogares energéticamente eficientes (Marmolejo-Duarte, García-Hooghuis, \& Masià, 2020).

La paridad de red depende del territorio (pues es una función de los costos de la tecnología), del perfil de consumo eléctrico del hogar, y de la reglamentación sobre el tratamiento que se da a la electricidad que el SFV genera, pero que no es consumida por el hogar. Esta situación tendrá repercusiones en las dinámicas de las ciudades, las cuales son objeto de estudio actual.

Una revisión de la literatura publicada en la base de datos Scopus® en el período 2014-2019 con la ecuación de búsqueda (1), arroja 46 resultados útiles para capturar la literatura científica, publicada alrededor de la evaluación financiera de la generación de electricidad solar para hogares:

(1) TITLE((pvs OR solar) AND (house* OR resident* OR home) AND (finance* OR economic))

Estas evaluaciones se han llevado a cabo para diferentes países, como se presenta en la Figura 1.

Figura 1. Aplicaciones de las investigaciones

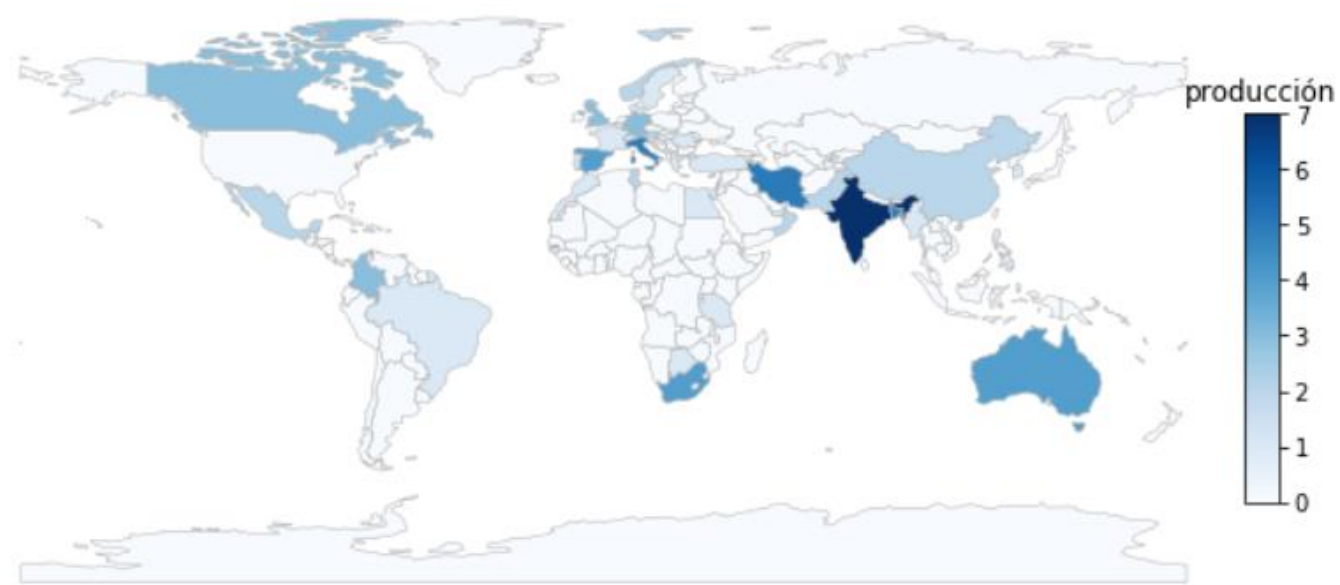

Fuente: elaboración propia, a partir de información de Scopus®

ACE, 15 (4.3) CC BY-ND 3.0 ES | UPC Barcelona, España | Las ciudades y el sol: Paridad de red de la generación de

electricidad con sistemas fotovoltaicos en los hogares en Colombia. DOI: http://dx.doi.org/10.5821/ace.15.43.8772

Cadavid, L.; Salazar, K.; Valencia, A; Franco, C. 
Las aplicaciones de las investigaciones son lideradas por Europa, con 37 investigaciones, seguidas por 29 en Asia, 12 en América y 10 en África. De las investigaciones en América, 8 han tenido como caso de aplicación algún territorio en Centro o Norte América, 3 abordan casos de Colombia y 1 aborda un caso en Brasil.

Puntualmente, para Colombia, estudios de evaluación de paridad residencial en el año 2014, indicaban que la viabilidad económica de la instalación de SFV en los hogares colombianos sería posible antes del año 2021, momento en el cual todas las ciudades del país alcanzarían la paridad de red (Jiménez, Cadavid, \& Franco, 2014). En el año 2015 y después de un fenómeno de devaluación intensa analizado en el año 2017 (Cadavid \& Franco, 2017), los resultados indicaban que la paridad dependía dramáticamente de la posibilidad de vender excedentes a la red eléctrica nacional (Cadavid, Jimenez, \& Franco, 2015). Estos estudios, fueron desarrollados cuando Colombia aún no había reglamentado las ventas de los excedentes de generación solar de los hogares, a la red eléctrica nacional, de manera que los excedentes no eran económicamente retribuidos a los hogares.

Sin embargo, en el año 2014, el gobierno colombiano promulgó el Acto de Energía Renovable (Congreso de la República de Colombia, 2014), cuyo propósito es promover el desarrollo y uso de fuentes de energía no convencionales para la generación de electricidad. Como resultado de este acto, fue posible para un usuario residencial, llevar a cabo actividades de microgeneración de electricidad para autoconsumo, haciendo uso, por ejemplo, de un SFV. Este acto fue formalizado en el año 2018 y desde entonces, la regulación colombiana permite a los hogares vender los excesos de electricidad de su SFV a la red eléctrica nacional, a un precio reglamentado por la Resolución CREG 30 de 2018 (Comisión de Regulación de Energía y Gas [CREG], 2018). Este precio es diferencial, es decir, existe un precio de venta que opera cuando la cantidad de electricidad vendida a la red, es inferior a la cantidad de electricidad comprada a la red por el hogar, y otro precio que opera cuando la electricidad vendida es superior a la electricidad comprada. De esta manera, los análisis son sensibles a la demanda de electricidad de los hogares, de un lado, y al tamaño de la solución solar, del otro lado.

La nueva reglamentación colombiana no involucra subsidios para la adquisición de los paneles solares. Tradicionalmente, los subsidios se han reconocido como un mecanismo efectivo para la promoción de esta tecnología a nivel residencial, como en el caso de Reino Unido (Ambrose, 2019; The Economic Times, 2019). Sin embargo, en otros casos, los subsidios han resultado perjudiciales para el desarrollo de las tecnologías renovables. A gran escala, se cree que los subsidios pausan la innovación y desestimulan la inversión de grandes jugadores (Bhushal, 2019); a pequeña escala, una administración inadecuada de los planes de subsidios puede llevar a crecimientos acelerados del mercado y a fallas en el mismo, como es el caso de España (Terrasa \& Di Lolli, 2017). Además, al masificar la tecnología a nivel residencial, los subsidios desincentivan la inversión en nueva capacidad en la red nacional; capacidad que sería necesaria en las noches para los sistemas solares que no usan baterías, creando así, dinámicas aún inciertas en el sistema eléctrico nacional (Potter \& Macdonald-Smith, 2019). Lo cierto, es que no es claro el impacto de los subsidios en la difusión de estas tecnologías, y los países adelantan medidas para evaluar las consecuencias de estos mecanismos en los territorios (De Miguel, 2018).

Colombia es un país con una capacidad instalada de generación de 16,78 GW, que suple una demanda de 66,67 TWh, en donde el sector residencial es el principal sector consumidor de electricidad, con el 42\% del consumo total (Unidad de Planeación Minero Energética [UPME], 2016).La generación depende principalmente de fuentes hídricas (86\% de la generación) y plantas térmicas (13\% de la generación) (XM, 2017). A pesar del gran potencial que tiene el país para generar electricidad a partir de fuentes renovables como la eólica, la solar, la biomasa o a través de mini-hidroeléctricas, estas alternativas no han sido los suficientemente exploradas en Colombia y las grandes hidroeléctricas y plantas térmicas dominan los planes actuales de expansión.

ACE, 15 (4.3) CC BY-ND 3.0 ES | UPC Barcelona, España | Las ciudades y el sol: Paridad de red de la generación de 
Estas circunstancias y el cambio en la reglamentación colombiana (que no involucra subsidios), genera nuevas preguntas: ¿Está Colombia en un estado de paridad de red, considerando la nueva normatividad que establece el precio de venta de los excedentes de la generación residencial?, ¿Qué capacidad debería tener un SFV residencial para alcanzar los máximos beneficios económicos?

\subsection{Métricas de evaluación}

Los análisis financieros para evaluar la implementación de un SVF, pueden llevarse a cabo a través de varias metodologías, y los resultados pueden resumirse utilizando diferentes indicadores. Por ejemplo, es posible calcular la suma simple del costo total, el costo promedio para un kWh generado, el valor presente neto de los flujos de efectivo del proyecto solar, la tasa interna de retorno, el período de recuperación y algunos otros indicadores, para poder concluir que los SFV han alcanzado la paridad de red para los hogares. De hecho, la literatura muestra que no existe un consenso sobre qué indicador usar para evaluar el costo de implementar un SFV para uso residencial. Aunque un solo indicador puede proporcionar información importante a los inversionistas o tomadores de decisiones sobre la implementación de un SFV, cuando la decisión requiere una comparación entre más de una opción de inversión, el análisis puede mejorarse considerando más de un indicador (López-Dumrauf, 2010; Ross, Westerfield, \& Jordan, 2010). La Tabla 1 presenta los indicadores usados en algunas de las investigaciones recientes.

Tabla 1. Indicadores de paridad

\begin{tabular}{|c|c|c|c|c|c|c|}
\hline Autores & Año & VPN & LCOE & PR-PRD & TIR & País \\
\hline Al-Saqlawi et al. & 2018 & $x$ & & $x$ & $x$ & Omán \\
\hline Korsavi et al. & 2018 & $x$ & $x$ & $x$ & & Irán \\
\hline Kim et al. & 2018 & $x$ & & & $x$ & Birmania \\
\hline Nyholm et al. & 2017 & & & & & Suecia \\
\hline Zandi et al. & 2017 & $x$ & & $x$ & $x$ & Irán \\
\hline Shahzad et al.. & 2017 & $x$ & $x$ & $x$ & & Pakistán \\
\hline Hazami et al. & 2017 & & & $x$ & & Túnez \\
\hline Alsharif & 2017 & $x$ & & & & Corea del Sur \\
\hline Akter et al. & 2017 & $x$ & $x$ & $x$ & $x$ & Australia \\
\hline Rodrigues et al. & 2017 & $x$ & & $x$ & $x$ & China \\
\hline Numbi and Malinga & 2017 & & & $x$ & & Suráfrica \\
\hline Linssen et al. & 2017 & $x$ & & & & Alemania \\
\hline Tomar and Tiwari & 2017 & $x$ & $x$ & & & Nueva Delhi \\
\hline Razmjoo et al.. & 2017 & $x$ & $x$ & $x$ & & Irán \\
\hline Quoilin et al. & 2016 & & $x$ & & & $\begin{array}{l}8 \text { países de la Unión } \\
\text { Europea y Reino Unido }\end{array}$ \\
\hline Assunção et al. & 2016 & $x$ & & $x$ & & Portugal \\
\hline Halder & 2016 & $x$ & & & $x$ & Bangladesh \\
\hline Cucchiella et al. & 2016 & $x$ & & & & Italia \\
\hline Zubi et al. & 2016 & $x$ & $x$ & & & $\begin{array}{l}\text { India, Pakistán, Tanzania y } \\
\text { España }\end{array}$ \\
\hline Cadavid and Franco & 2017 & & $x$ & & & Colombia \\
\hline Cadavid et al. & 2015 & & $x$ & & & Colombia \\
\hline Grande, et al. & 2015 & $x$ & & $x$ & & México \\
\hline Kantor et al. & 2015 & $x$ & & & & Canadá \\
\hline Lang et al. & 2015 & & & & $x$ & Alemania, Suiza y Austria \\
\hline Jiménez et al. & 2014 & & $x$ & & & Colombia \\
\hline Holdermann et al. & 2014 & $x$ & & & & Brasil \\
\hline Abu-Bakar et al. & 2014 & $x$ & & & $x$ & Reino Unido \\
\hline
\end{tabular}

Nota: VPN: Valor Presente Neto. LCOE: Costo Nivelado de la Electricidad. PR: Periodo de Recuperación. PRD: Período de Recuperación Descontado. TIR: Tasa Interna de Retorno. CAE: Costo Anual Equivalente. Fuente: elaboración propia, con base en la bibliografía mencionada.

ACE, 15 (43) CC BY-ND 3.0 ES | UPC Barcelona, España Las ciudades y el sol: Paridad de red de la generación de 
Puede notarse que, el VPN es una medida ampliamente usada en las investigaciones de paridad de red solar residencial (Akter, Mahmud, \& Oo, 2017; Al-Saqlawi, Madani, \& Mac Dowell, 2018; Alsharif, 2017; Assunção, Moura, \& de Almeida, 2016; Axaopoulos \& Fylladitakis, 2013; Cucchiella, D’Adamo, \& Gastaldi, 2016; Grande, Islas, \& Rios, 2015; Halder, 2016; Holdermann, Johannes, \& BeigelJürgen, 2014; Kantor, Rowlands, Parker, \& Lazowski, 2015; Kim \& Jung, 2018; Razmjoo, Qolipour, Shirmohammadi, Heibati, \& Faraji, 2017; Rodrigues, Chen, \& Morgado-Dias, 2017; Shahzad et al., 2017; Zubi, Dufo-López, Pardo, \& Pasaoglu, 2016). Esta medida involucra los costos asociados con la compra, reemplazo y mantenimiento de los equipos, considerando el momento en que estos eventos ocurren durante todo el ciclo de vida del proyecto; posteriormente, los valores son traídos a valor presente usando una tasa de descuento del dinero o costo de oportunidad (Richard, Myers, \& Allen, 2017). En efecto, el VPN es el indicador más usado para evaluar proyectos de inversión (Graham \& Harvey, 2002) y para algunos autores, es el que debe prevalecer cuando otros indicadores arrojan resultados contradictorios (Berk \& Demarzo, 2008).

El LCOE, empleado por autores como Akter, Mahmud y Oo, (2017) Cadavid, Jimenez y Franco (2014; 2015) Halder (2016), Razmjoo et al. (2017), Shahzad et al. (2017), Tomar y G.N. Tiwari (2017), Korsavi, Zomorodian y Tahsildoost (2018) y Quoilin et al. (2016a), es el segundo indicador con mayor uso en la literatura para la evaluación de la paridad de red solar residencial. Este indicador usa el concepto del VPN para calcular los costos por kWh generados por el SFV, dividiendo el VPN entre la electricidad generada durante la vida útil del proyecto. Existen diferentes versiones para el cálculo del LCOE, algunas de las cuales descuentan la electricidad generada con una tasa de descuento (Short, Packey, \& Holt, 1995), y otras proponen la división sin descontar la electricidad (HOMER Energy, 2018). Para que un proyecto de generación solar residencial sea considerado beneficioso, el LCOE debe ser inferior a la tarifa de la red eléctrica o bien, inferior al costo actual al que se compra la electricidad que usa el hogar.

Por su estructura de cálculo, el comportamiento del LCOE es idéntico al comportamiento del VPN para la evaluación de la paridad de red, permitiendo así, llegar a las mismas conclusiones de viabilidad del proyecto. Sin embargo, el LCOE arroja resultados en términos de costo por kWh generado, en lugar de costos totales del proyecto como lo hace el VPN. Por esta razón, en este estudio, el análisis de costos se realiza empleando el LCOE.

El PR es otro método de evaluación empleado por varios autores (Akter et al., 2017; Al-Saqlawi et al., 2018; Grande et al., 2015; Halder, 2016; Hazami et al., 2017; Korsavi et al., 2018; Razmjoo et al., 2017; Shahzad et al., 2017; Suárez, Prieto, \& Fernández, 2013; Zandi et al., 2017). Para su cálculo se requiere que haya flujos de caja positivos. En el caso de la instalación de un SFV en el cual se tienen sólo flujos negativos (costos de generación), es necesario considerar una segunda alternativa con los costos de comprar electricidad a la red eléctrica nacional; la comparación entre esas dos alternativas, permite construir un flujo de caja diferencial, a través del cual se determina el tiempo que tarda en recuperarse la inversión en el SFV.

Una variación del PR es el PRD (Akter et al., 2017; Assunção et al., 2016; Numbi \& Malinga, 2017; Rodrigues et al., 2017), que reconoce el valor del dinero en el tiempo para el cálculo del período de recuperación. La literatura sugiere el uso del PRD para análisis más conservadores que con el PR. Para que un proyecto de generación solar residencial sea considerado beneficioso, el PRD debe ser inferior al plazo de evaluación.

Finalmente, la TIR es otro indicador usado para los análisis de paridad (Abu-Bakar et al., 2014; Akter et al., 2017; Halder, 2016; Kim \& Jung, 2018; Lang, Gloerfeld, \& Girod, 2015; Rodrigues et al., 2017; Zandi et al., 2017). La TIR es la tasa de descuento de los flujos de caja que hace que valor presente neto de costos (compra, mantenimiento y reemplazo) y beneficios (ahorros y ventas de electricidad a la red)

ACE, 15 (4.3) CC BY-ND 3.0 ES | UPC Barcelona, España | Las ciudades y el sol: Paridad de red de la generación de 6 electricidad con sistemas fotovoltaicos en los hogares en Colombia. DOI: http://dx.doi.org/10.5821/ace.15.43.8772

Cadavid, L.; Salazar, K.; Valencia, A; Franco, C. 
sea cero, es decir, que hace que los costos sean iguales a los beneficios. Para que un proyecto de generación solar residencial sea considerado beneficioso, la TIR debe ser mayor al costo de capital del hogar (Ross, Westerfield, \& Jaffe, 2012).

En estudios previos, otros autores han evaluado la paridad de red en diferentes ciudades, empleando modelos financieros en los cuales proyectan los flujos de caja para periodos de evaluación entre 20 y 25 años. Estos flujos, comúnmente consideran, los costos de la inversión, del reemplazo de los equipos, de los mantenimientos y, según el caso, las compras y ventas de energía al sistema. Autores como Akter, Mahmud y Oo (2017) analizan los ahorros en el pago a la empresa de servicios públicos, para SFV de diferentes capacidades con y sin baterías, considerando la estructura tarifaria en Australia. Estudios similares, se han llevado a cabo en países en vía de desarrollo como Irán, en donde Korsavi, Zomorodian y Tahsildoost (2018), concluyeron que los sistemas fotovoltaicos sólo son viables bajo esquemas de tarifas sin subsidios. En India (Tomar \& G.N. Tiwari, 2017), Portugal, Rumania, Italia, Dinamarca y Grecia (Quoilin et al., 2016), igualmente se evaluó la viabilidad de instalar SFV en hogares. En estas investigaciones, los resultados, indicaron que sólo las instalaciones sin baterías y que se encuentren conectadas a la red, permitían recuperar la inversión en tiempos (entre 6 y 22 años) menores al periodo de evaluación. Además, en ninguno de los casos se encontró que fuera viable económicamente bajo las condiciones estudiadas, satisfacer el 100\% de la demanda. Es común que en estos estudios se considere una configuración arbitraria de paneles y baterías, que puede ser óptima o no, para la demanda del hogar. Asimismo, es usual que la evaluación se aborde desde la perspectiva del consumo, sin considerar el costo de la producción de electricidad, aspectos que si son considerados en este trabajo.

En este artículo, se analiza la paridad de red solar residencial para Colombia, a través de 3 indicadores de evaluación: LCOE, TIR y PRD. Para ello, se desarrolla un modelo financiero que proyecta los flujos de caja de la instalación de un SFV, y los compara con el costo de comprar la electricidad a la red nacional. La configuración del SFV responde a una capacidad de generación y almacenamiento óptima en términos económicos, para cada nivel de atención de la demanda. Adicionalmente, se calcula la reducción de dióxido de carbono en la generación de electricidad, al implementar el sistema fotovoltaico.

El caso de Colombia es interesante por dos razones: (1) es un país que se encuentra en la zona ecuatorial, de manera que se espera que la generación solar sea favorable en términos económicos; (2) desde el año 2018, la regulación permite a los hogares, la venta de los excedentes de electricidad que se generen con el SFV, a la red eléctrica nacional. Por lo tanto, los hogares se preparan para esta nueva manera de adherirse al mercado de electricidad nacional.

\section{Metodología}

\subsection{Herramienta de evaluación}

Para validar la paridad de la red en Colombia, se desarrolló un modelo que proyecta mensualmente los flujos de caja de la instalación de un sistema fotovoltaico, en un hogar promedio de una ciudad en Colombia. Los flujos de caja de un sistema fotovoltaico (que, en adelante, se señala cómo el escenario Solar), son comparados con los flujos de caja del hogar, cuando se compra la energía eléctrica a la red nacional (el cual, para propósito de este análisis, será llamado el escenario Red).

En la Figura 2 se presenta un esquema de un SFV residencial, así como ejemplos de instalaciones de SFV en hogares en Colombia.

ACE, 15 (4.3) CC BY-ND 3.0 ES | UPC Barcelona, España | Las ciudades y el sol: Paridad de red de la generación de 


\section{ACE Architecture, City and Environment}

Figura 2. Ejemplos de instalaciones de SFV en hogares en Colombia

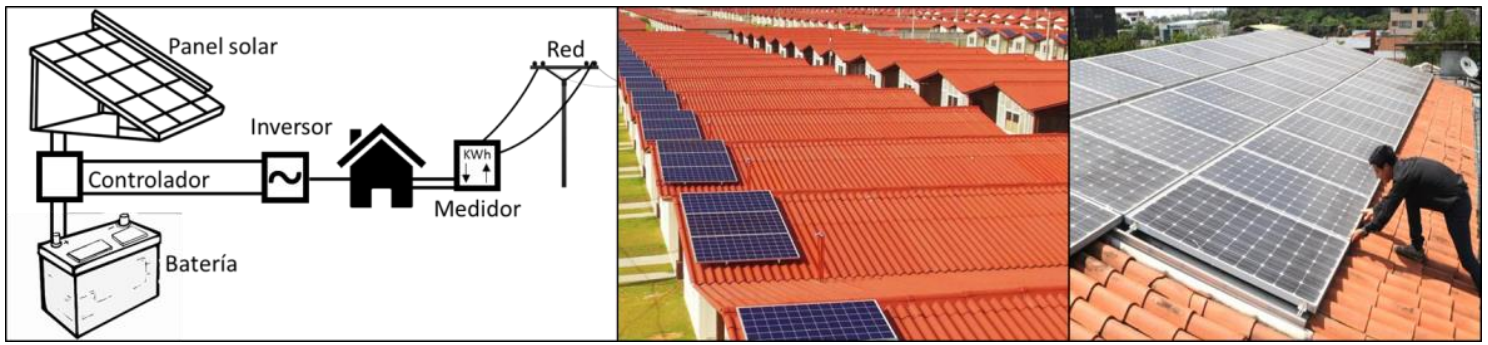

Fuente: A: Adaptación de Luthander et al. (2015) B: fotografía de Celsia (2019). C: fotografía de El País (2018)

En la Figura 3A se observa la configuración clásica de un SVF. Los paneles solares se conectan con las baterías a través del regulador de carga, que controla la carga de las baterías desde los paneles y la descarga de las mismas hacia el circuito eléctrico de la vivienda. El controlador se conecta al inversor de corriente, que permite la conversión de la corriente directa en corriente alterna, para que pueda ser empleada por los receptores y electrodomésticos utilizados en la vivienda. La vivienda, a su vez, está conectada a la red eléctrica nacional a través de un medidor de electricidad que hace un conteo de la electricidad consumida de la red y de la electricidad entregada por el hogar a la red. En las figuras 3B y 3C, se presentan fotografías de una instalación típica de paneles en techos de viviendas colombianas, tanto en un conjunto residencial (3B) como en una vivienda independiente (3C).

Para la evaluación financiera de las alternativas, se emplearon tres indicadores: el LCOE (ahorro porcentual del LCOE del escenario Solar respecto al LCOE del escenario Red), la TIR y el PRD. Estos dos últimos indicadores han sido calculados considerando los flujos de caja incrementales, como resultado de comparar Red vs Solar. Para determinar si se alcanza la paridad de la red, el porcentaje de ahorro del LCOE Solar frente al LCOE de la Red, debe ser positivo. Para determinar la paridad con la TIR, esta debe ser superior al costo de capital del hogar, y en el caso del PRD, este debe ser inferior al plazo estipulado por el hogar.

El modelo considera un horizonte de evaluación de 20 años. La elección del período de evaluación tuvo en cuenta dos aspectos: (1) el periodo de garantía ofrecido por los proveedores de los equipos en el mercado colombiano (Ecolumen Soluciones Energéticas, 2018; Erco Energía, 2018), que corresponde a 20 años. Y (2) lo reportado en investigaciones similares, como las desarrolladas por Ramadhan y Naseeb (2011), Linssen Stenzel y Fleer (2017), Al-Saqlawi, Madani y Mac Dowell (2018), y Kim y Jung (2018), en las cuales se han usado periodos de evaluación de 20 años o superiores. Autores como Shahzad (2017), Akter, Mahmud y Oo (2017), y Korsavi, Zomorodian y Tahsildoost (2018), sugieren un período de evaluación de 25 años.

La configuración óptima del SFV para satisfacer la demanda del hogar, es un resultado obtenido con el modelo. En él, se varía el número de paneles y baterías (de 0 a 12), hasta encontrar la combinación con el costo mínimo para un determinado nivel de demanda. Este costo se determina según el indicador LCOE, tal como como lo hacen los sistemas SAM (National Renewable Energy Laboratory [NREL], 2017) y HOMER (HOMER Energy, 2018). Para las configuraciones del SFV, los flujos de caja consideran la inversión inicial en la solución solar (que incluye la compra de los paneles solares, las baterías, el inversor, el cableado, los soportes y la instalación), los reemplazos del inversor y las baterías, así como los costos de mantenimiento de los equipos (usualmente, asociados con limpieza). Dependiendo del tamaño y la configuración de la solución solar, es posible que el hogar necesite comprar alguna electricidad a la red nacional, o que genere excedentes que pueda vender a la red. De esta manera, el modelo considera las compras y las ventas de electricidad a la red, según sea el caso, dependiendo de la cantidad de energía generada por el sistema fotovoltaico y la electricidad demandada por el hogar. 
Aunque los flujos de caja son mensuales, la generación de electricidad fue calculada por horas para un día típico. La electricidad producida por la alternativa solar y disponible para usar por el hogar en un instante (t), depende de la generación solar y la capacidad de almacenamiento en el año específico. La generación solar en el instante ( $\mathrm{t}$ ), depende de la capacidad del sistema fotovoltaico y su tasa de degradación. Esta es calculada con la ecuación (2):

$$
\text { (2) } \quad S E_{t}=C s *\left(1-s d_{t}\right)^{t} * C f_{t}
$$

Donde (SEt) es la generación solar o electricidad producida por el SFV en el instante (t); (Cs) es la capacidad instalada del sistema; $\left(s d_{t}\right)$ es la tasa de degradación en el instante $(\mathrm{t})$; ( $\left.c f_{t}\right)$ es el factor de carga o brillo solar disponible en el lugar de la instalación, en el instante (t).

La capacidad de almacenamiento de las baterías depende de su tasa de degradación para el año específico. Se asume que las baterías inician el día descargadas y la carga final es calculada para cada hora en un día típico, según la generación solar y la demanda en el instante (t), haciendo un balance de inventario de carga según la ecuación (3):

$$
C h_{t}=\min \left[C h_{t-1}+S E_{t}-D_{t}, \operatorname{Cbg} \times\left(1-b d_{t}\right)^{t}\right]
$$

Donde ( $\mathrm{Ch}_{\mathrm{t}}$ ) es la carga de la batería en el instante (t); ( $\left.\mathrm{Ch}_{\mathrm{t}-1}\right)$ es la carga de la batería en el instante $(t-1) ;\left(D_{t}\right)$ es la demanda de electricidad en el instante (t); es la capacidad instalada de la batería; $\left(b_{t}\right)$ es la tasa de degradación de la batería en el instante (t).

Para el caso base de análisis, los indicadores de evaluación fueron calculados considerando la electricidad demandada por el hogar (en adelante, ec), la cual puede ser tomada de su SFV o de la red nacional (en el caso en el cual el SFV genere y almacene una cantidad de electricidad insuficiente para satisfacer la demanda). En este sentido, los flujos de caja consideran las compras necesarias a la red nacional cuando el SFV es muy pequeño para cubrir la demanda, y se dividen los gastos e ingresos del proyecto solar entre la energía demandada por el hogar. Esta metodología es la empleada por Homer Software (HOMER Energy, 2018) y la aplicada en algunos casos de estudio (Alghamdi, Castro, \& Zamora, 2018; Das, Tan, Yatim, \& Lau, 2017; Ghenai, Salameh, \& Merabet, 2018; Mansur, Baharudin, \& Ali, 2017; Shahzad et al., 2017). Los análisis considerando la ec, son útiles para evaluar la viabilidad económica de un SFV (dado el perfil del hogar y las reglamentaciones del territorio). Sin embargo, para efectos de evaluación tecnológica, es útil analizar los costos de producción de la electricidad con el SFV. En este tipo de análisis, el cálculo de los indicadores ignora la compra de energía a la red cuando la solución solar no satisface el 100\% de la demanda del hogar. En cambio, se dividen los gastos e ingresos del proyecto solar entre la energía generada por los paneles, sin importar si esta fue usada en el hogar o vendida a la red. Esta metodología, es útil para determinar si en términos técnicos, la tecnología produce o no, electricidad más barata que la electricidad de la red, sin importar el uso que se le dé a la misma (uso en el hogar o uso para venta). Con este fin, se evaluaron también los indicadores considerando el total de la electricidad solar producida por la solución (en adelante, ep). La ep puede ser inferior a la ec si el SFV tiene poca capacidad de generación, o ser superior, cuando el SFV tiene una alta capacidad de generación.

Adicionalmente, se calculó la reducción de emisiones de $\mathrm{CO}_{2}$, comparando los $\mathrm{KgCO}_{2}$ que se dejan de emitir al emplear el SFV, con las emisiones que produce la generación de electricidad en el sistema nacional. El análisis se hizo a nivel individual (hogar) y colectivo (ciudad).

La evaluación económica de los sistemas solares fotovoltaicos depende de las condiciones del territorio, por lo cual se hace necesaria la elección de un caso de aplicación.

ACE, 15 (4.3) CC BY-ND 3.0 ES | UPC Barcelona, España | Las ciudades y el sol: Paridad de red de la generación de 


\subsection{Caso de aplicación}

Gracias a su diversidad climática, Colombia cuenta con ciudades con alto brillo solar que oscila entre 4,4 y 7,7 horas diarias promedio (Instituto de Hidrología Meteorología y Estudios Ambientales [IDEAM], 2016). Dentro de estas ciudades, Medellín cuenta con 5,1 horas diarias promedio de brillo solar intenso (Sistema Único de Información de Servicios Públicos [SUI], 2018), de manera que puede considerarse una ciudad promedio en el territorio (ni muy brillante ni muy oscura). Además, es la segunda ciudad más grande del país (si se considera toda su área Metropolitana), albergando cerca de 4 millones de habitantes (alrededor del 10\% de la población nacional) (Departamento Administrativo Nacional de Estadística [DANE], 2018c).

Por esas razones, se eligió como caso de aplicación un hogar promedio en la ciudad de Medellín. Esta ciudad, registró alrededor de 892.000 unidades de vivienda en el año 2018, de las cuales el 50,9\% corresponde a casas y el 46,1\% corresponde a apartamentos (Departamento Administrativo Nacional de Estadística [DANE], 2018a). En promedio, en una casa (cuyo área promedio es de 149 m2) habitan 4,1 personas, mientras que en un apartamento (con un área promedio de $74 \mathrm{~m} 2$ ) habitan 3,5 personas (Dinero, 2019).

Con base en las características físicas del inmueble y su entorno, las viviendas se encuentran clasificadas en 6 estratos socioeconómicos. Los estratos 1, 2 y 3 corresponden a estratos bajos que albergan a los usuarios con menores recursos, los cuales son beneficiarios de subsidios en la tarifa de la electricidad (50\% para el estrato 1, 40\% para el estrato 2 y 15\% para el estrato 3). Los estratos 5 y 6 , corresponden a estratos altos que albergan a los usuarios con mayores recursos económicos, los cuales deben pagar una contribución adicional del $20 \%$ sobre el valor de la tarifa de la electricidad para auxiliar a los estratos 1,2 y 3. El estrato 4 no es beneficiario de subsidios ni debe pagar sobrecostos, sino que paga exactamente el valor que la empresa defina como costo de la electricidad (Departamento Administrativo Nacional de Estadística [DANE], 2018c). La Figura 3 presenta la distribución de los hogares en función del estrato socioeconómico en Medellín.

Figura 3. Distribución de los hogares por estrato en Medellín

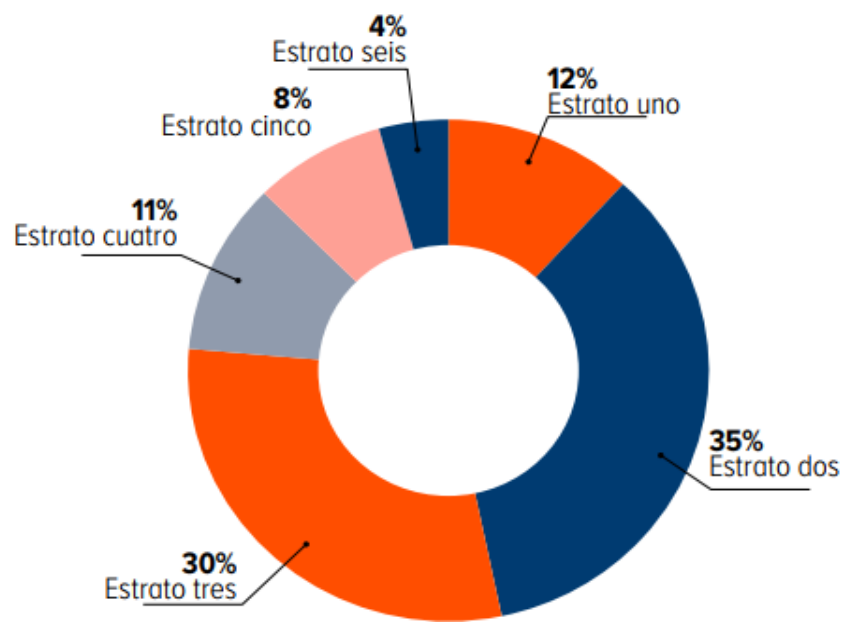

Fuente: Medellín como vamos (2018) datos del Departamento Administrativo de Planeación con base en instalaciones Empresas Públicas de Medellín

Se observa que el 77\% de las viviendas están en los estratos 1, 2 o 3, mientras que el 23\% restante se encuentra en los estratos 4, 5 y 6. Asimismo, los estratos se caracterizan por diferentes niveles de consumo de electricidad. El estrato 1 consume en promedio $101 \mathrm{kWh}$ al mes, mientras que el estrato 2 consume 123 kWh mensuales. Los estratos 3, 4, 5 y 6 consumen 140, 165, 198, y 275 kWh al mes, respectivamente (datos de Empresas Públicas de Medellín, en (Trujillo, 2017)). 
Debido a que la tarifa pagada por el usuario del estrato 4 no se afecta por subsidios ni contribuciones adicionales, se eligió para el análisis, una vivienda de estrato 4 en Medellín. A este estrato pertenecen alrededor de 98.000 viviendas de la ciudad, las cuales consumen un promedio de $165 \mathrm{kWh}$ mensuales. En este estrato, más del $70 \%$ de las viviendas del país usan la electricidad para sus servicios de iluminación, así como para hacer uso del televisor, la licuadora, la nevera, la plancha, el reproductor de DVD, la lavadora, el computador, el equipo de sonido, y efectuar la carga del teléfono móvil. Otros estratos, sin embargo, presentan una tenencia distinta de electrodomésticos, como se presenta en la Tabla 2.

Tabla 2. Tenencia porcentual de electrodomésticos por estrato en Colombia

\begin{tabular}{|l|l|l|l|l|l|l|}
\hline Electrodoméstico & Estrato 1 & Estrato 2 & Estrato 3 & Estrato 4 & Estrato 5 & Estrato 6 \\
\hline Iluminación & $100 \%$ & $100 \%$ & $100 \%$ & $100 \%$ & $100 \%$ & $100 \%$ \\
\hline Televisor & $93 \%$ & $96 \%$ & $89 \%$ & $100 \%$ & $100 \%$ & $100 \%$ \\
\hline Celular & $80 \%$ & $88 \%$ & $83 \%$ & $100 \%$ & $100 \%$ & $100 \%$ \\
\hline Licuadora & $81 \%$ & $85 \%$ & $79 \%$ & $93 \%$ & $90 \%$ & $100 \%$ \\
\hline Refrigeración & $75 \%$ & $87 \%$ & $83 \%$ & $100 \%$ & $100 \%$ & $100 \%$ \\
\hline Plancha & $66 \%$ & $76 \%$ & $69 \%$ & $79 \%$ & $100 \%$ & $100 \%$ \\
\hline DVD & $57 \%$ & $66 \%$ & $69 \%$ & $71 \%$ & $80 \%$ & $100 \%$ \\
\hline Lavadora & $50 \%$ & $67 \%$ & $75 \%$ & $86 \%$ & $100 \%$ & $100 \%$ \\
\hline Computador & $41 \%$ & $52 \%$ & $70 \%$ & $93 \%$ & $100 \%$ & $100 \%$ \\
\hline Equipo de sonido & $49 \%$ & $47 \%$ & $50 \%$ & $71 \%$ & $90 \%$ & $100 \%$ \\
\hline
\end{tabular}

Fuente: Elaboración propia, con base en (Unidad de Planeación Minero Energética \& Corpoema-Cusa, 2011).

La Tabla 3 presenta los parámetros empleados en el modelo con respecto al caso de aplicación para un hogar en esta ciudad. Las fuentes de información de estos parámetros son primarias o de fuentes certificadas, sin embargo, el modelo desarrollado corresponde a un caso hipotético de evaluación, como es usual encontrarlos en la literatura para este tipo de análisis.

Tabla 3. Parámetros del modelo

\begin{tabular}{|c|c|c|}
\hline Parámetro & Valor & Fuente \\
\hline Costo del panel solar* & $1,13[\$ / W]$ & $\begin{array}{l}\text { (Ambiente Soluciones, 2018; Ecolumen } \\
\text { Soluciones Energéticas, 2018; Erco Energía, } \\
\text { 2018; Hersic International, 2018; Hybrytec S.A.S., } \\
\text { 2018; Solarplus, 2018) }\end{array}$ \\
\hline Costo del inversor* & $0,29[\$ / W]$ & $\begin{array}{l}\text { (Ambiente Soluciones, 2018; Ecolumen } \\
\text { Soluciones Energéticas, 2018; Erco Energía, } \\
\text { 2018; Hersic International, 2018; Hybrytec S.A.S., } \\
\text { 2018; Solarplus, 2018) }\end{array}$ \\
\hline Costo de las baterías* & $0,5[\$ / W]$ & $\begin{array}{l}\text { (Ambiente Soluciones, 2018; Ecolumen } \\
\text { Soluciones Energéticas, 2018; Erco Energía, } \\
\text { 2018; Hersic International, 2018; Hybrytec S.A.S., } \\
\text { 2018; Solarplus, 2018) }\end{array}$ \\
\hline $\begin{array}{l}\text { Tasa de degradación del } \\
\text { SFV }\end{array}$ & $\begin{array}{l}\text { Lineal, considerando eficiencia } \\
\text { de } 0,8 \text { al final de su vida útil }\end{array}$ & (Branker, Pathak, \& Pearce, 2011) \\
\hline $\begin{array}{l}\text { Tasa de degradación de } \\
\text { las baterías }\end{array}$ & $\begin{array}{l}\text { Lineal, considerando eficiencia } \\
\text { de } 0,6 \text { al final de su vida útil }\end{array}$ & $\begin{array}{l}\text { (Kyriakopoulos \& Arabatzis, 2016; Luo, Wang, } \\
\text { Dooner, \& Clarke, 2015) }\end{array}$ \\
\hline $\begin{array}{l}\text { Factor de carga de la } \\
\text { ciudad }\end{array}$ & 0,2117 & $\begin{array}{l}\text { (Instituto de Hidrología Meteorología y Estudios } \\
\text { Ambientales [IDEAM], 2016) }\end{array}$ \\
\hline $\begin{array}{l}\text { Demanda promedio de un } \\
\text { hogar** }\end{array}$ & $165[\mathrm{kWh} / \mathrm{mes}]$ & $\begin{array}{l}\text { (Sistema Único de Información de Servicios } \\
\text { Públicos [SUI], 2018) }\end{array}$ \\
\hline $\begin{array}{l}\text { Tarifa promedio de } \\
\text { electricidad red nacional }\end{array}$ & $0,16[\$ \cup S D / k W h]$ & $\begin{array}{l}\text { (Sistema Único de Información de Servicios } \\
\text { Públicos [SUI], 2018) }\end{array}$ \\
\hline $\begin{array}{l}\text { Precio de venta de los } \\
\text { excedentes del SFV }\end{array}$ & $\begin{array}{l}0,15[\$ \cup S D / k W h] \text { para ventas } \\
\text { inferior a compras. } \\
\text { 0,04 [\$USD/kWh] para ventas } \\
\text { superiores a compras }\end{array}$ & $\begin{array}{l}\text { (Comisión de Regulación de Energía y Gas } \\
\text { [CREG], 2018) }\end{array}$ \\
\hline
\end{tabular}

Nota: *El valor de los equipos considera el precio de venta local del equipo después de costos de importación. ** La demanda de electricidad del hogar típico se considera fija para todo el horizonte de evaluación.

Fuente: Elaboración propia partir de las fuentes citadas. 
Para la inversión inicial, se consideró una tecnología fotovoltaica de celdas monocristalinas y un inversor con eficiencia del 98\% y una vida útil de 10 años, dado que son las tecnologías más comunes para la generación solar en Colombia y que los estudios prueban su idoneidad para suplir las demandas residenciales (Zalamea-León, Mena-Campos, Moscoso-Cordero, Barragán-Escandón, \& Méndez-Santos, 2018). Se escogieron baterías de litio con una vida útil estimada de 12 años, como lo proponen Kyriakopoulos y Arabatzis (2016), al igual que Luo et al. (2015). Los flujos de caja consideran el remplazo del inversor y de las baterías, después de 10 y 12 años de operación respectivamente. Tanto los costos de mantenimiento como las compras y las ventas a la red, son calculados mensualmente en el modelo.

\section{Resultados}

Los resultados obtenidos para el caso base se presentan en la Figura 4. El eje horizontal presenta diferentes configuraciones del SFV en términos de capacidad de generación (G) [kW] y capacidad de almacenamiento (A) [kW]. En el eje vertical primario, se representan la TIR y los ahorros porcentuales en el LCOE. En el eje vertical secundario, se presentan los años que tarda el proyecto en recuperar la inversión.

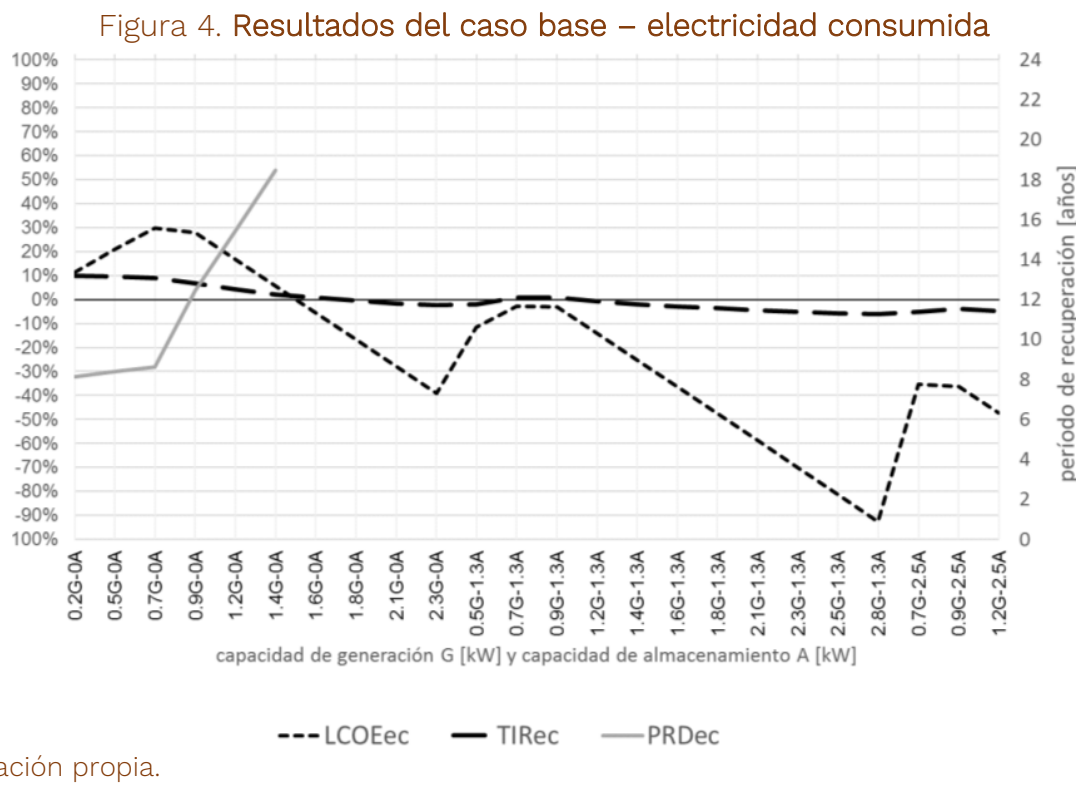

Fuente: elaboración propia.

Con los resultados obtenidos es posible afirmar que el territorio se encuentra en condiciones de paridad. Las primeras seis configuraciones evaluadas en la Figura 4, son viables y pueden satisfacer entre el 29\% y 49\% de la electricidad que consume el hogar con autoproducción y consumo directo. Estos porcentajes, se encuentran por encima del cubrimiento promedio de la demanda para un hogar en Europa, el cual se ubica entre el 30\% y 37\% (Quoilin et al., 2016), con un SFV sin baterías. Para las demás instalaciones, el valor de la inversión hace que el costo de la electricidad sea mayor, comparado con el del sistema interconectado nacional. La combinación con mejores resultados corresponde a una solución con 3 paneles y cero baterías $(0,7 \mathrm{~kW}$ y OkW), la cual representa un ahorro del 30\% en el costo de la electricidad para el hogar, un LCOE de 0,11\$USD/kWh, una TIR de 9,1\% y un periodo de recuperación de la inversión de 8,5 años. Esta solución, permitiría abastecer la demanda del hogar en un 46\%. Las ventas realizadas a la red en esta solución representan un 40,6\% de la energía consumida. Entre las configuraciones viables, la de menor ahorro (6\%), permite cubrir un 49\% de la demanda con 6 paneles, cuya inversión se recupera en 18 años y presenta un LCOE de $0,15 \$ \cup S D / k W h$ con una TIR de 2,2\%. 
Para las instalaciones que generan ahorros, se obtuvieron periodos de recuperación entre $8(0,23$ kW) y 18 años (1,38 kW). Al contrastar con estudios previos, Zandi et al (2017a) obtuvieron periodos entre 6 y 17 años para su mejor escenario analizado (sin baterías), Akter, Mahmud y Oo (2017), encontraron que para instalaciones con baterías, los periodos de recuperación iban desde 22 años hasta superar los 35 años, excediendo el tiempo de evaluación de 25 años; mientras que para instalaciones de sólo paneles, el rango de tiempo según la capacidad, variaba entre 10 y 15 años. De manera similar, Korsavi, Zomorodian y Tahsildoost (2018) encontraron PRD promedio de 12 años para tarifas sin subsidios, y entre 47 y 50 años para tarifas subsidiadas.

Nótese que los ahorros disminuyen cuando se hace inversión en almacenamiento. Es decir que, aunque los sistemas fotovoltaicos con baterías, permiten un mayor cubrimiento de la demanda con la electricidad que genera el SFV, el costo de los equipos no se compensa con las menores compras, ni con las ventas que se realizan a la red.

Estos resultados, pueden compararse contra los obtenidos suponiendo que la venta de excedentes de electricidad de los hogares a la red nacional, no fuera posible (Figura 5), es decir, la situación antes de la implementación de la resolución CREG 30, que entró en vigencia en el año 2018.

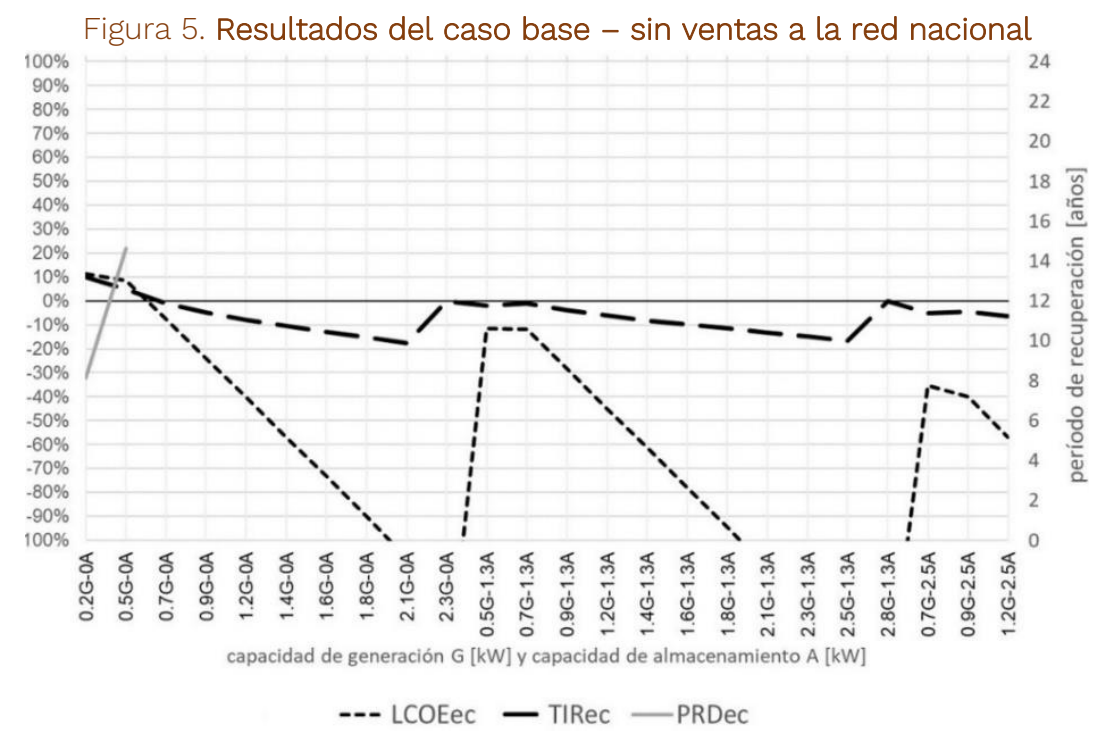

Fuente: elaboración propia.

Sin la reglamentación que permite la venta de excedentes a la red, el máximo ahorro posible es del $10 \%$, obtenido con una configuración con capacidad de generación de $0,2 \mathrm{~kW}$ sin baterías y que satisface el 44\% de la demanda. En términos de costos, bajo este escenario, el LCOE de esta solución representa 0,15\$USD/kWh para una configuración con un panel y cero baterías, cuya inversión se recupera en 8 años. La otra configuración económicamente viable, logra un ahorro del 5\% con dos paneles (0,5KWh) sin baterías y un cubrimiento del $29 \%$ de la demanda, para un LCOE de 0,14\$USD/kWh. Las demás configuraciones, no son económicamente viables en ausencia de la reglamentación y conforme se agrega mayor capacidad de generación, el costo solar se hace más alto. Resultados similares se encontraron para el caso australiano, en donde instalaciones con baterías que no están conectadas a la red, no son viables y solo el SFV sin baterías y de menor capacidad, es viable, con un periodo de recuperación de 15 años (Akter et al., 2017).

Volviendo al escenario actual, en el que la reglamentación del año 2018 permite la venta de excedentes a la red nacional, los resultados de la simulación considerando la electricidad producida, se presentan en la Figura 6. 


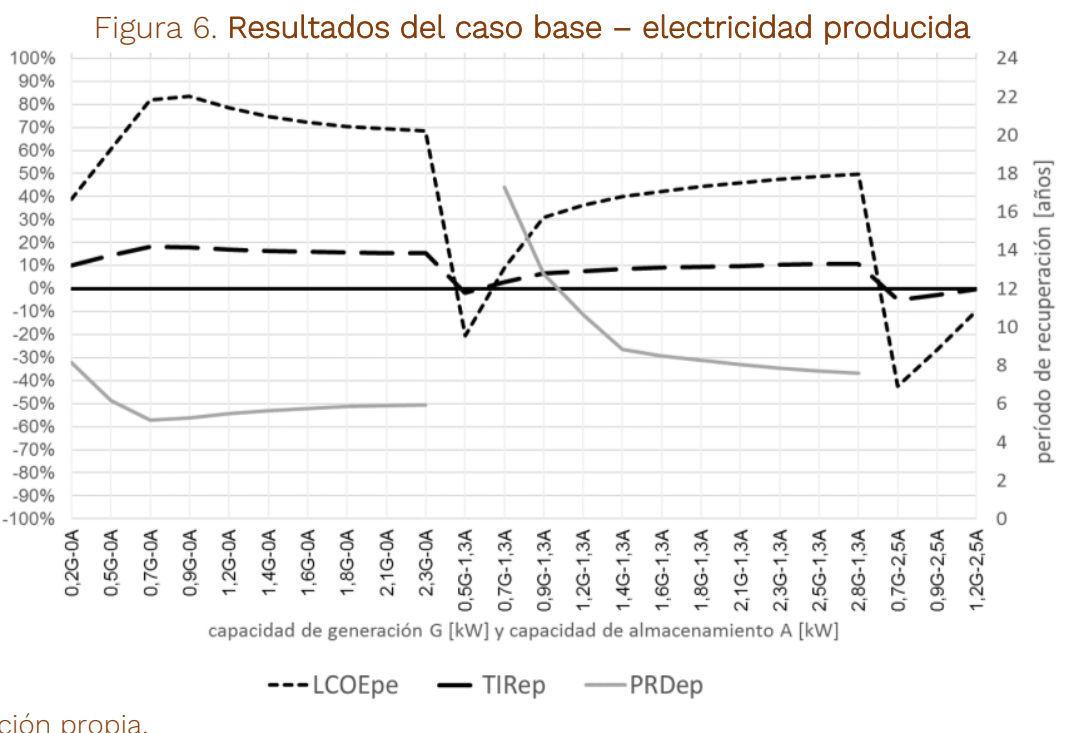

Fuente: elaboración propia.

Desde el punto de vista de la electricidad producida, 20 configuraciones del SFV muestran ahorros (14 más que en el escenario ec). La configuración que genera los mayores ahorros corresponde a 0,9 KWh (4 panales) y representa un LCOE de 0,02 \$USD/kWh, con un cubrimiento de 47\% de la demanda. Las soluciones sin baterías con capacidad de generación entre 0,2 kW y 2,3 kW, así como aquellas de entre $0,7 \mathrm{~kW}$ y 2,8 kW de generación y almacenamiento de 1,3 kW, permiten generar energía más barata que la que puede obtenerse comprando a la red. Estas configuraciones representan ahorros entre 9\% y 84\% para el hogar, permiten la recuperación de la inversión en menos de 8 años para las soluciones sin batería, y entre 8 y 18 años para las soluciones con alguna capacidad de almacenamiento. Asimismo, abastecen de forma directa entre el $29 \%$ y $80 \%$ de la electricidad demandada por el hogar.

Dada la reducción esperada de los costos de la tecnología, la evaluación también se realizó considerando que la inversión se realizaría en el año 2025. Según las proyecciones de la Agencia Internacional de Energías Renovables (IRENA, 2019), en el año 2025 el precio de los equipos podría ser aproximadamente un 70\% del precio en 2018. Para las baterías, se estima que haya disminuido al 75\% (IRENA, 2017). Los resultados teniendo en cuenta la electricidad consumida para el año 2025 se presentan en la Figura 7.

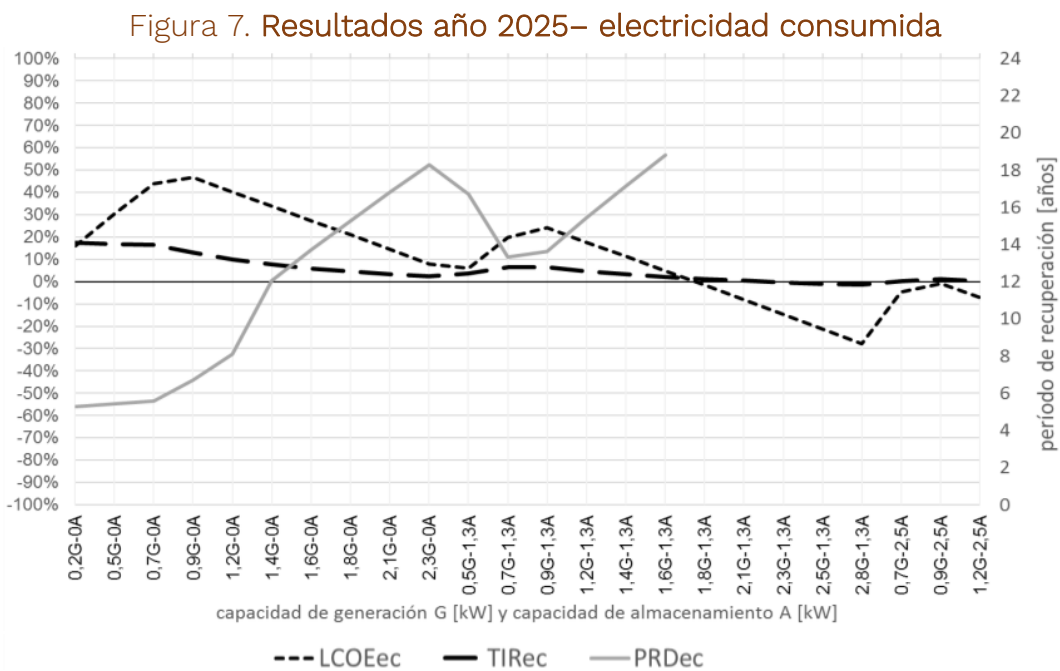

Fuente: elaboración propia.

ACE, 15 (4.3) CC BY-ND 3.0 ES | UPC Barcelona, España Las ciudades y el sol: Paridad de red de la generación de 
Debido a la disminución de los costos de inversión en el año 2025, 16 configuraciones (10 más que en el año 2018) serían económicamente factibles, generando así, ahorros para el hogar entre el 5\% y el $47 \%$ en su costo de electricidad. La configuración óptima tendría una capacidad de 0,9kW de generación (sin almacenamiento), alcanzando la recuperación de la inversión en 6,7 años y una TIR del 13,2\%. La demanda atendida con este SFV sería del 47\% y el LCOE sería 0,09\$USD/kWh.

Debido a que soluciones con mayor capacidad de generación y almacenamiento empiezan a ser económicamente factibles, es posible que hasta el $76 \%$ de la demanda sea cubierta con la electricidad generada y consumida directamente del SFV, representando ahorros para el hogar incluso en este caso. Los resultados obtenidos, están alineados con los de investigaciones anteriores (Akter et al., 2017; Quoilin et al., 2016; Tomar \& G.N. Tiwari, 2017; Zubi et al., 2016), en donde se identifica que la inversión en baterías, si bien permite disminuir el LCOE conforme se dispone de mayor cantidad de energía para consumir o vender a la red (con el enfoque pe), se requiere que el costo de estas, siga disminuyendo para hacer viables las instalaciones con mayor capacidad instalada y que permitan cubrir porcentajes de la demanda más altos desde el punto de vista de ce. Igualmente, se obtienen mejores resultados cuando el SFV se encuentra conectado a la red nacional, que cuando no lo está (Akter et al., 2017; Tomar \& G.N. Tiwari, 2017).

La paridad de red no sólo permite generar ahorros para los hogares en el costo de la energía eléctrica que consumen, sino también disminuir el impacto medioambiental de la huella de carbono, al reemplazar las tecnologías de generación por unas más limpias. Para el caso colombiano, la UPME (2016), ha estimado que el consumo de $1 \mathrm{KWh}$ del Sistema Interconectado Nacional representa una emisión de 0,199 $\mathrm{KgCO}_{2}$. Cabe mencionar, que las emisiones dependen de las características de la matriz energética del país; al ser la hídrica la mayor fuente de generación en Colombia, estas emisiones pueden estar incluso, un 70\% por debajo de otros cálculos a nivel internacional, como los sugeridos por el Departamento de Medio Ambiente del Gobierno de Aragón (n.d.) (0,40 $\mathrm{KgCO}_{2}$ ), la iniciativa española de CeroCO2 (n.d.) $\left(0,41 \mathrm{KgCO}_{2}\right)$ o la U.S. Environmental Protection Agency- EPA (2018) $\left(0,71 \mathrm{KgCO}_{2}\right)$. Esto quiere decir, que un hogar promedio colombiano que satisface el $49 \%$ de su demanda con un SFV, podría ahorrar en un año $193 \mathrm{KgCO}_{2}$ de emisiones. A nivel de ciudad el impacto sería mayor; por ejemplo, si se considera un escenario en el que el 50\% de los hogares en estratos 4 , 5 y 6 (aquellos con mayores consumos por hogar y con mayor capacidad adquisitiva), generan energía con un SFV bajo las condiciones del caso base, sería posible ahorrar $23.500 \mathrm{TonCO}_{2}$ en emisiones anuales, lo que significaría una reducción del 24,5\% en las emisiones de $\mathrm{CO}_{2}$ que genera el $23 \%$ de la población de Medellín.

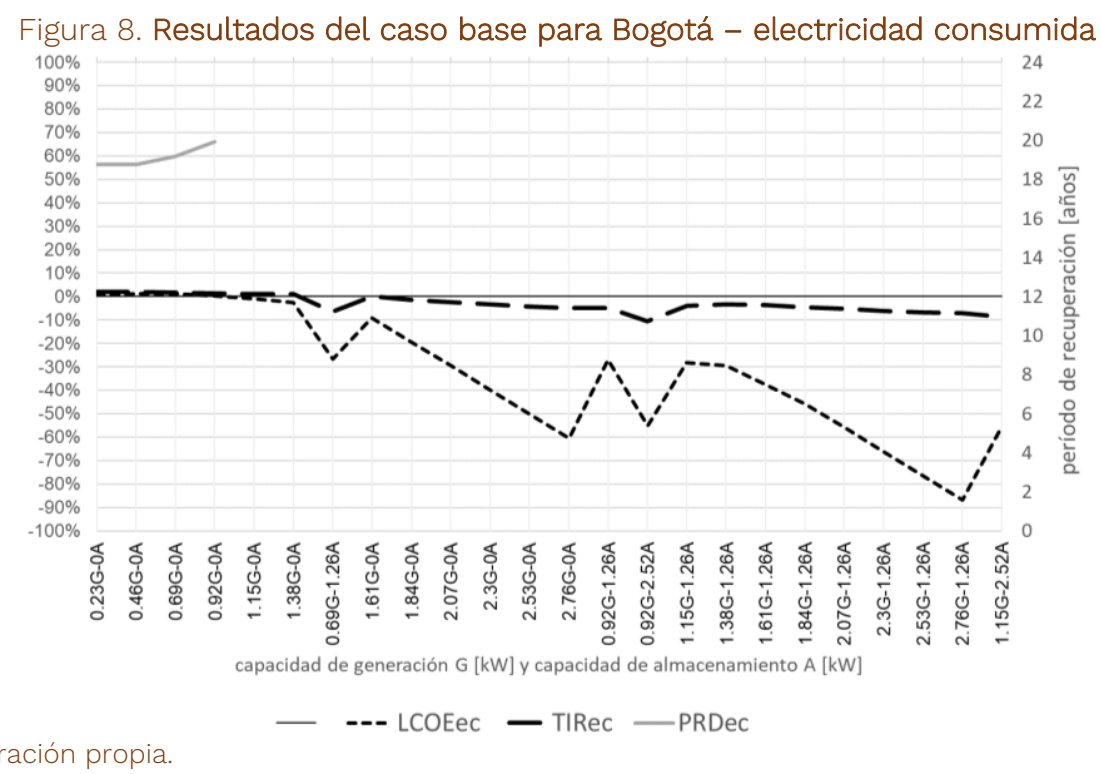

Fuente: elaboración propia.

ACE, 15 (4.3) CC BY-ND 3.0 ES | UPC Barcelona, España | Las ciudades y el sol: Paridad de red de la generación de 
Los hallazgos reportados son extrapolables a territorios con condiciones similares de radiación solar y tarifas de electricidad. Sin embargo, territorios con condiciones diferentes pueden llevar a comportamientos distintos. Por ejemplo, en la Figura 8 se presentan los resultados del caso base para la ciudad de Bogotá, la capital de Colombia que alberga cera de 7,2 millones de personas (Departamento Administrativo Nacional de Estadística [DANE], 2018c), en alrededor de 2,5 millones de hogares. Los hogares de Bogotá, tienen un consumo mensual promedio de 176 kWh y pagan un precio de 0,11 \$/kWh por la electricidad de la red eléctrica nacional. La ciudad tiene un factor de carga solar de 0,182 (Instituto de Hidrología Meteorología y Estudios Ambientales [IDEAM], 2016), que corresponde a 4,3 horas de brillo solar intenso diario.

Bogotá se encuentra en condiciones de paridad. Las primeras cuatro configuraciones evaluadas (que corresponden a 1, 2, 3 y 4 paneles, sin baterías) son viables, y pueden satisfacer entre el 15\% y $45 \%$ de la electricidad que consume el hogar con autoproducción y consumo directo. Sin embargo, estas soluciones aún no representan ahorros significativos para el hogar, respecto al valor que pagarían consumiendo la electricidad desde la red eléctrica nacional, y los periodos de recuperación de la inversión superan los 18 años. Es decir, que si bien el precio de la electricidad de la red eléctrica nacional para Bogotá es superior al precio para Medellín (así como el consumo de electricidad de cada hogar) y ello podría implicar un mayor potencial para la instalación de soluciones solares residenciales, la menor intensidad de brillo solar de la ciudad termina afectando negativamente este potencial, llevando a que las soluciones solares sean menos beneficiosas para Bogotá que para Medellín, en términos de ahorro para los hogares.

De acuerdo con la Encuesta Nacional de Calidad de Vida (ECV) de Colombia, Medellín y Bogotá, tienen una calidad de vida similar (8,5 para la primera y 8,3 para la segunda, en una escala sobre 10) (Departamento Administrativo Nacional de Estadística [DANE], 2018b)). Es decir, que ambas ciudades comparten, entre otras, características socioeconómicas e infraestructurales. Las implicaciones de estas características en la evaluación económica de la paridad solar y, por tanto, las implicaciones de las diferencias de estas características entre ciudades en la paridad de red, requieren análisis distintos que se encuentran por fuera del objetivo perseguido en esta investigación.

\section{Conclusiones}

Actualmente, Medellín (Colombia) se encuentra en paridad de red para atender hasta un $49 \%$ de la demanda de un hogar típico con electricidad que proviene directamente de SFV para autoconsumo, adquirido a precio de mercado sin subsidios. La configuración óptima en el análisis, fue aquella con una capacidad instalada de generación de $0,7 \mathrm{~kW}$ sin baterías y que cubre el $49 \%$ de la demanda. A través de esta configuración, se genera un ahorro del 30\% en el costo de la electricidad para el hogar, una TIR de 9,1\%, y se recupera la inversión realizada en el sistema fotovoltaico en 8,5 años.

Los resultados positivos, son posibles gracias a la política de venta de los excedentes de generación de los hogares a la red nacional, que entró en vigencia en el año 2018. En ausencia de esta política, el ahorro máximo sería del 10\% para una configuración más pequeña (de 0,5kW sin baterías). De esta manera, es posible afirmar que la reglamentación de venta de excedentes permitió a los hogares un ahorro adicional del $20 \%$ en su factura de electricidad bajo un esquema de autogeneración solar.

En el año 2025, es posible atender una mayor porción de la demanda a través de la generación de electricidad con un SFV, de una manera costo efectiva, satisfaciendo hasta el 79\% de la demanda y a la vez, generando ahorros. Los ahorros máximos en el año 2025 son del 47\% del costo de la factura, 17\% más que en el año 2018, con una configuración de 0,9kW sin baterías y que cubre el 47\% de la demanda. 
El valor de la inversión en almacenamiento de electricidad en el hogar, a través de baterías, disminuye los ahorros a medida que aumenta la capacidad de almacenamiento. Es decir, aunque los sistemas fotovoltaicos con baterías permiten un mayor cubrimiento de la demanda, el costo de los equipos no se compensa con las menores compras que se realizan a la red. En este sentido, la red actúa como un mecanismo de almacenamiento que resulta menos costoso que las baterías, aún incluso, considerando la venta de excedentes a la red.

Cuando el análisis se hace teniendo en cuenta la electricidad producida por los paneles y no la demandada por el hogar, configuraciones que no alcanzan la paridad bajo el enfoque de la electricidad consumida, lo hacen desde el enfoque de la electricidad producida. Es decir, este último enfoque es favorecido en la evaluación financiera, aunque no refleja la realidad económica de la factura de electricidad de los hogares, sino que se centra en el costo de producción.

La implementación de energías limpias permite la reducción de emisiones de dióxido de carbono en la generación de electricidad. A mayor capacidad del SFV, mayor será la reducción. En Medellín, podrían ahorrarse cerca de 24.000 toneladas anuales de $\mathrm{CO}_{2}$, si el $50 \%$ de los hogares en los estratos socioeconómicos 4, 5 y 6, implementan un sistema que atienda hasta el 49\% de su demanda.

Estos resultados son extrapolables a territorios con condiciones similares de radiación solar y tarifas de electricidad, aunque la metodología puede replicarse en territorios con condiciones diferentes.

\section{Agradecimientos}

Al Instituto Tecnológico Metropolitano de Medellín, Colombia por su apoyo para la publicación. Los autores también agradecen a los revisores sus valiosos aportes y comentarios, los cuales contribuyeron al perfeccionamiento del trabajo presentado.

\section{Autoría}

La primera autora desarrolló el modelo de evaluación. La segunda autora y el tercer autor construyeron el marco teórico y el análisis de resultados. El cuarto autor contribuyó en la idea original del proyecto y validó los resultados. Todos los autores participaron activamente en la elaboración del documento escrito.

Conflicto de intereses: Los autores declaran que no hay conflicto de intereses.

\section{Bibliografía}

Abu-Bakar, S.H.; Muhammad-Sukki, F.; Ramirez-Iniguez, R; Munir, A.B.; Mohd Yasin, S.H.; Mallick, T.K., ... Abdul Rahim, R. (2014). Financial analysis on the proposed renewable heat incentive for residential houses in the United Kingdom: A case study on the solar thermal system. Energy Policy, 65, 552-561. DOI: https://doi.org/10.1016/J.ENPOL.2013.10.018

Akter, M.N.; Mahmud, M.A., \& Oo, A.M.T. (2017). Comprehensive economic evaluations of a residential building with solar photovoltaic and battery energy storage systems: An Australian case study. Energy and Buildings, 138, 332-346. DOI: https://doi.org/10.1016/j.enbuild.2016.12.065

Al-Saqlawi, J.; Madani, K., \& Mac Dowell, N. (2018). Techno-economic feasibility of grid-independent residential roof-top solar PV systems in Muscat, Oman. Energy Conversion and Management, 178, 322334. DOI: https://doi.org/10.1016/J.ENCONMAN.2018.10.021 
Alghamdi, A.H.S.; Castro, C.H.M.R., \& Zamora, R. (2018). Review of Cost Optimization of Electricity Supply by Using HOMER and a Case Study for a Big Commercial Customer in Brazilian Amazon Area. DOI: https://doi.org/10.1007/978-3-319-94965-9 22

Alsharif, M.H. (2017). Optimization design and economic analysis of energy management strategy based on photovoltaic/energy storage for heterogeneous cellular networks using the HOMER model. Solar Energy, 147, 133-150. DOI: https://doi.org/10.1016/j.solener.2017.03.044

Ambiente Soluciones. (2018). Productos - Energía solar. Recuperado de https://www.ambientesoluciones.com/sitio/productos lineas.php?c=409

Ambrose, J. (2019, June 5). Home solar panel installations fall by $94 \%$ as subsidies cut. The Guardian. Recuperado de https://www.theguardian.com/environment/2019/jun/05/home-solar-panelinstallations-fall-by-94-as-subsidies-cut

Assunção, A.; Moura, P.S., \& de Almeida, A.T. (2016). Technical and economic assessment of the secondary use of repurposed electric vehicle batteries in the residential sector to support solar energy. Applied Energy, 181, 120-131. DOI: https://doi.org/10.1016/j.apenergy.2016.08.056

Axaopoulos, P.J., \& Fylladitakis, E. D. (2013). Performance and economic evaluation of a hybrid photovoltaic/thermal solar system for residential applications. Energy and Buildings, 65, 488-496. DOI: https://doi.org/10.1016/j.enbuild.2013.06.027

Bazilian, M.; Onyeji, I.; Liebreich, M.; MacGill, I.; Chase, J.; Shah, J., ... Zhengrong, S. (2013). Re-considering the economics of photovoltaic power. Renewable Energy, 53, 329-338. DOI: https://doi.org/10.1016/j.renene.2012.11.029

Berk, J., \& Demarzo, P. (2008). Corporate finance (1st ed.). Mexico: Pearson Education Inc.

Bhandari, R., \& Stadler, I. (2009). Grid parity analysis of solar photovoltaic systems in Germany using experience curves. Solar Energy, 83(9), 1634-1644. DOI: https://doi.org/10.1016/j.solener.2009.06.001

Bhushal, R. (2019, August 5). Subsidies killing renewable energy investments in Nepal. TheThirdPole. Recuperado de https://www.thethirdpole.net/en/2019/08/05/subsidies-killing-renewable-energyinvestments-in-nepal/

Branker, K., Pathak, M. J. M., \& Pearce, J. M. (2011, December). A review of solar photovoltaic levelized cost of electricity. Renewable and Sustainable Energy Reviews, 15, 4470-4482. DOI: https://doi.org/10.1016/j.rser.2011.07.104

Breyer, C., \& Gerlach, A. (2013). Global overview on grid-parity. Progress in Photovoltaics: Research and Applications, 21(1), 121-136. DOI: https://doi.org/10.1002/pip.1254

Cadavid, L., \& Franco, C.J. (2017). Impact of devaluation on grid parity for residential solar generation in Latin America. IEEE América Latina, 15(11). DOI: https://doi.org/10.1109/TLA.2017.8070414

Cadavid, L.; Jimenez, M., \& Franco, C. J. (2015). Financial analysis of photovoltaic configurations for Colombian households. IEEE Latin America Transactions, 13(12), 3832-3837. DOI: https://doi.org/10.1109/TLA.2015.7404916

Celsia. (2019). Celsia lanza línea de solución de energía solar para hogares. Recuperado de https://www.celsia.com/es/sala-prensa/celsia-lanza-l237nea-de-soluci243n-de-energ237a-solarpara-hogares-1

Centre for Alternative Technology [CAT]. (2019). Photovoltaic (PV) Solar Panels. Recuperado de December 23, 2019, https://www.cat.org.uk/info-resources/free-information-service/energy/solarphotovoltaic/

Ceroco2. (n.d.). Cálculo de Huella de Carbono por consumo eléctrico. Recuperado de https://www.ceroco2.org/calculadoras/

Comisión de Regulación de Energía y Gas [CREG]. (2018). Resolución 030 de 2018. Recuperado de http://apolo.creg.gov.co/Publicac.nsf/1c09d18d2d5ffb5b05256eee00709c02/83b41035c2c4474f05258 243005a1191?OpenDocument 
Congreso de la República de Colombia. (2014). Ley 1715 de 2014: integración de las energías renovables no convencionales al Sistema Energético Nacional. Recuperado de http://www.secretariasenado.gov.co/senado/basedoc/ley 1715 2014.html

Cucchiella, F.; D’Adamo, I., \& Gastaldi, M. (2016). Photovoltaic energy systems with battery storage for residential areas: an economic analysis. Journal of Cleaner Production, 131, 460-474. DOI: https://doi.org/10.1016/j.jclepro.2016.04.157

Das, H.S.; Tan, C.W.; Yatim, A.H.M., \& Lau, K.Y. (2017). Feasibility analysis of hybrid photovoltaic/battery/fuel cell energy system for an indigenous residence in East Malaysia. Renewable and Sustainable Energy Reviews, 76, 1332-1347. DOI: https://doi.org/10.1016/J.RSER.2017.01.174

De Miguel, B. (2018, February 1). La UE auditará las ayudas a las renovables en España. El País. Recuperado de https://cincodias.elpais.com/cincodias/2018/02/01/companias/1517503408 651759.html

Departamento Administrativo Nacional de Estadística [DANE]. (2018a). Censo Nacional de Población y Vivienda 2019. Recuperado de https://sitios.dane.gov.co/cnpv/\#!/como vivimos

Departamento Administrativo Nacional de Estadística [DANE]. (2018b). Encuesta nacional de calidad de vida. Recuperado de https://www.dane.gov.co/index.php/estadisticas-por-tema/salud/calidadde-vida-ecv/encuesta-nacional-de-calidad-de-vida-ecv-2018

Departamento Administrativo Nacional de Estadística [DANE]. (2018c). Estratos socioeconómicos Colombia. Recuperado https://www.dane.gov.co/files/geoestadistica/Preguntas frecuentes estratificacion.pdf

Departamento de Medio Ambiente del Gobierno de Aragón. (n.d.). Calculadora de emisiones de CO2. Recuperado de http://calcarbono.servicios4.aragon.es/

Deutsche Bank report. (2015). Solar grid parity in a low oil price era. Recuperado de June 24, 2019, https://www.db.com/cr/en/concrete-deutsche-bank-report-solar-grid-parity-in-a-low-oil-priceera.htm

Dinero. (2019, May 15). Apartamentos en Colombia tienen mayor demanda que casas. Dinero. Recuperado de https://www.dinero.com/pais/articulo/apartamentos-en-colombia-tienen-mayordemanda-que-casas/272006

Ecolumen Soluciones Energéticas. (2018). Productos - páneles solares. Recuperado de December 4, 2017. Recuperado de http://www.ecolumen.net/productos.php

El País. (2018). Caleños podrán producir y vender energía solar, ¿cómo hacerlo? Recuperado de December 23, 2019, El País. Website: https://www.elpais.com.co/contenido-premium/calenospodran-producir-y-vender-energia-solar-como-hacerlo.html

Erco Energía. (2018). Servicios - nuestras soluciones. Recuperado de http://www.ercoenergia.com.co

Ghenai, C.; Salameh, T., \& Merabet, A. (2018). Technico-economic analysis of off grid solar PV/Fuel cell energy system for residential community in desert region. International Journal of Hydrogen Energy, 45(20), 11460-11470. DOI: https://doi.org/10.1016/J.IJHYDENE.2018.05.110

Graham, J., \& Harvey, C. (2002). How Do Cfo S Make Capital Budgeting and Capital Structure. Journal of Applied Corporate Finance, 15(1), 8-23. DOI: https://doi.org/10.1111/j.1745-6622.2002.tb00337.x

Grande, G.; Islas, J., \& Rios, M. (2015). Technical and economic analysis of Domestic High Consumption Tariff niche market for photovoltaic systems in the Mexican household sector. Renewable and Sustainable Energy Reviews, 48, 738-748. DOI: https://doi.org/10.1016/J.RSER.2015.04.038

Halder, P.K. (2016). Potential and economic feasibility of solar home systems implementation in Bangladesh. Renewable and Sustainable Energy Reviews, 65, 568-576. DOI: https://doi.org/10.1016/j.rser.2016.07.062 
Hazami, M.; Mehdaoui, F.; Naili, N.; Noro, M.; Lazzarin, R., \& Guizani, A. (2017). Energetic, exergetic and economic analysis of an innovative Solar CombiSystem (SCS) producing thermal and electric energies: Application in residential and tertiary households. Energy Conversion and Management, 140, 36-50. DOI: https://doi.org/10.1016/j.enconman.2017.02.040

Hersic International. (2018). Portafolio - energía fotovoltaica. Recuperado de https://www.hersic.com

Holdermann, C.; Kissel, J., \& Beigel, J. (2014). Distributed photovoltaic generation in Brazil: An economic viability analysis of small-scale photovoltaic systems in the residential and commercial sectors. Energy Policy, 67, 612-617. DOI: https://doi.org/10.1016/j.enpol.2013.11.064

HOMER Energy. (2018). Levelized Cost of Energy. Recuperado de January 9, 2018, https://www.homerenergy.com/support/docs/3.11/total electrical load served.html

Hybrytec S.A.S. (2018). Hybrytec empresa colombiana lider en energía solar. Recuperado de https://www.hybrytec.com/

Instituto de Hidrología Meteorología y Estudios Ambientales [IDEAM]. (2016). Promedios Climatológicos 1981-2010. Recuperado de Características Climatológicas de Colombia website: http://www.pronosticosyalertas.gov.co/mapas-graficos-tiempo-clima/indicadores-climatologicos

International Energy Agency, I. (2018). Market Report Series: Renewables 2018 - English ES. In International Energy Agency. Recuperado de IEA Publications website: https://webstore.iea.org/market-report-series-renewables-2018

IRENA. (2017). Electricity storage and renewables: Costs and markets to 2030. In Lpublications/2017/Oct/Electricity-storage-and-renewables-costs-and-markets. Recuperado de https://www.irena.org/publications/2017/Oct/Electricity-storage-and-renewables-costs-and-markets

IRENA. (2019). Renewable Energy Statistics 2019. In International Renewable Energy Agency (Vol. 1). Recuperado de https://www.irena.org/publications/2019/Jul/Renewable-energy-statistics-2019

Jiménez, M.; Cadavid, L., \& Franco, C. (2014). Scenarios of photovoltaic grid parity in Colombia. Dyna, 188(81), 237-245. DOI: https://doi.org/10.15446/dyna.v81n188.42165

Kantor, I.; Rowlands, I.H.; Parker, P., \& Lazowski, B. (2015). Economic feasibility of residential electricity storage systems in Ontario, Canada considering two policy scenarios. Energy and Buildings, 86, 222232. DOI: https://doi.org/10.1016/J.ENBUILD.2014.10.022

Kim, H., \& Jung, T.Y. (2018). Independent solar photovoltaic with Energy Storage Systems (ESS) for rural electrification in Myanmar. Renewable and Sustainable Energy Reviews, 82, 1187-1194. DOI: https://doi.org/10.1016/J.RSER.2017.09.037

Korsavi, S.S.; Zomorodian, Z.S., \& Tahsildoost, M. (2018). Energy and economic performance of rooftop PV panels in the hot and dry climate of Iran. Journal of Cleaner Production, 174, 1204-1214. DOI: https://doi.org/10.1016/J.JCLEPRO.2017.11.026

Kyriakopoulos, G.L., \& Arabatzis, G. (2016). Electrical energy storage systems in electricity generation: Energy policies, innovative technologies, and regulatory regimes. Renewable and Sustainable Energy Reviews, 56, 1044-1067. DOI: https://doi.org/10.1016/J.RSER.2015.12.046

Lang, T.; Gloerfeld, E., \& Girod, B. (2015). Don't just follow the sun - A global assessment of economic performance for residential building photovoltaics. Renewable and Sustainable Energy Reviews, 42, 932-951. DOI: https://doi.org/10.1016/J.RSER.2014.10.077

Linssen, J.; Stenzel, P., \& Fleer, J. (2017). Techno-economic analysis of photovoltaic battery systems and the influence of different consumer load profiles. Applied Energy, 185, 2019-2025. DOI: https://doi.org/10.1016/j.apenergy.2015.11.088

López-Dumrauf, G. (2010). Finanzas corporativas: un enfoque latinoamericano (2d ed.). Argentina: Alfaomega.

ACE, 15 (43) CC BY-ND 3.0 ES | UPC Barcelona, España | Las ciudades y el sol: Paridad de red de la generación de 
Luo, X.; Wang, J.; Dooner, M., \& Clarke, J. (2015). Overview of current development in electrical energy storage technologies and the application potential in power system operation. Applied Energy, 137, 511-536. DOI: https://doi.org/10.1016/J.APENERGY.2014.09.081

Luthander, R.; Widén, J.; Nilsson, D., \& Palm, J. (2015). Photovoltaic self-consumption in buildings: A review. Applied Energy, 142, 80-94. DOI: https://doi.org/10.1016/J.APENERGY.2014.12.028

Mansur, T.M.N.T.; Baharudin, N.H., \& Ali, R. (2017). Optimal sizing and economic analysis of selfconsumed solar PV system for a fully DC residential house. 2017 IEEE 4th International Conference on Smart Instrumentation, Measurement and Application (ICSIMA), 1-5. DOI: https://doi.org/10.1109/ICSIMA.2017.8312006

Marmolejo-Duarte, C.; García-Hooghuis, A., \& Masià, A. G. (2020). How much and why are we willing to pay for energy-efficient homes? A stated preferences analysis in Barcelona. ACE: Architecture, City and Environment, 14(42). DOI: https://doi.org/10.5821/ace.14.42.9215

Medellincomovamos. (2018). Informe de calidad de vida Medellín 2018. Recuperado de https://www.medellincomovamos.org/download/documento-informe-de-calidad-de-vida-demedellin-2018/

Ministerio de Minas y Energía -MME-Unidad de Planeación Minero Energética - UPME. (2016). Calculadora Emisiones. In 2016.2 Recuperado de http://www.upme.gov.co/Calculadora Emisiones/aplicacion/calculadora.html

National Renewable Energy Laboratory [NREL]. (2017). System Advisor Model (SAM) |. Recuperado de January 18, 2018, https://sam.nrel.gov/

NC Sustainable Energy Association [NCSEA]. (2019). What is clean energy. Recuperado de December 15, 2019, https://energync.org/what-is-clean-energy/

Numbi, B.P., \& Malinga, S.J. (2017). Optimal energy cost and economic analysis of a residential gridinteractive solar PV system- case of eThekwini municipality in South Africa. Applied Energy, 186, 2845. DOI: https://doi.org/10.1016/j.apenergy.2016.10.048

Potter, B., \& Macdonald-Smith, A. (2019, October 12). Vic solar, fed interventions show failure of energy policy. Australian Financial Review. Recuperado de https://www.afr.com/politics/vic-solar-fedinterventions-show-failure-of-energy-policy-20181012-h16jw1

Quoilin, S.; Kavvadias, K.; Mercier, A.; Pappone, I., \& Zucker, A. (2016). Quantifying self-consumption linked to solar home battery systems: Statistical analysis and economic assessment. Applied Energy, 182, 58-67. DOI: https://doi.org/10.1016/j.apenergy.2016.08.077

Ramadhan, M., \& Naseeb, A. (2011). The cost benefit analysis of implementing photovoltaic solar system in the state of Kuwait. Renewable Energy, 36(4), 1272-1276. DOI: https://doi.org/10.1016/j.renene.2010.10.004

Razmjoo, A.; Qolipour, M.; Shirmohammadi, R.; Heibati, S.M., \& Faraji, I. (2017). Techno-economic evaluation of standalone hybrid solar-wind systems for small residential districts in the central desert of Iran. Environmental Progress \& Sustainable Energy, 36(4), 1194-1207. DOI: https://doi.org/10.1002/ep.12554

REN 21. (2019). Renewables 2019, Global Status Report (presentation). Recuperado de https://wedocs.unep.org/bitstream/handle/20.500.11822/28496/REN2019.pdf?sequence=1\&isAllowed =y\%OAhttp://www.ren21.net/cities/wp-content/uploads/2019/05/REC-GSR-Low-Res.pdf

Richard, B.; Myers, S., \& Allen, F. (2017). Principles of corporate finance.

Rodrigues, S., Chen, X., \& Morgado-Dias, F. (2017). Economic analysis of photovoltaic systems for the residential market under China's new regulation. Energy Policy, 101, 467-472. DOI: https://doi.org/10.1016/j.enpol.2016.10.039

Ross, S., Westerfield, R., \& Jaffe, J. (2012). Corporate finance (12th ed.). New York: Mc Graw Hill. 
Ross, S.; Westerfield, R., \& Jordan, B. (2010). Net present value and oter investment criteria. In Fundamentals of corporate finance (9th ed., pp. 260-288). New York: Mc Graw Hill.

Shahzad, M.K.; Zahid, A.; Rashid, T.; Rehan, M.A.; Ali, M., \& Ahmad, M. (2017). Techno-economic feasibility analysis of a solar-biomass off grid system for the electrification of remote rural areas in Pakistan using HOMER software. Renewable Energy, 106, 264-273. DOI: https://doi.org/10.1016/j.renene.2017.01.033

Short, W.; Packey, D.J., \& Holt, T. (1995). A Manual for the Economic Evaluation of Energy Efficiency and Renewable Energy Technologies. Colorado, USA: National Renewable Energy Laboratory [NREL].

Sistema Único de Información de Servicios Públicos [SUI]. (2018). Sistema Único de Información de Servicios Públicos [SUI]. Recuperado de http://superservicios.gov.co

Solarplus. (2018). Solar Plus - Planeación, asesoría, dirección, ejecución y control de proyectos de energía solar fotovoltaica y productos de consumo para la industria de este mercado. Recuperado de https://solarplusonline.com/

Suárez, I.; Prieto, M.M., \& Fernández, F. J. (2013). Analysis of potential energy, economic and environmental savings in residential buildings: Solar collectors combined with microturbines. Applied Energy, 104, 128-136. DOI: https://doi.org/10.1016/J.APENERGY.2012.10.070

Terrasa, R., \& Di Lolli, A. (2017, March 13). Viaje por la gran estafa solar. El Mundo. Recuperado de https://www.elmundo.es/economia/2017/03/13/58c11c3522601da3218b45e8.html

The Economic Times. (2019, May 15). Solar subsidies: Government subsidies and other incentives for installing rooftop solar system in India. The Economic Times. Recuperado de https://economictimes.indiatimes.com/small-biz/productline/power-generation/solar-subsidiesgovernment-subsidies-and-other-incentives-for-installing-rooftop-solar-system-inindia/articleshow/69338706.cms?from $=\mathrm{mdr}$

Tomar, V., \& G.N. Tiwari. (2017). Techno-economic evaluation of grid connected PV system for households with feed in tariff and time of day tariff regulation in New Delhi - A sustainable approach. Renewable and Sustainable Energy Reviews, 70, 822-835. DOI: https://doi.org/10.1016/J.RSER.2016.11.263

Trujillo, C. (2017). Estrato 6 del Aburrá consume más energía que la que debería. Recuperado de December 11, 2019, El Colombiano website: https://www.elcolombiano.com/antioquia/estrato-6consume-mas-energia-de-la-que-deberia-FA6651766

U.S. Energy Information Administration [EIA]. (2019). Capacity Factors for Utility Scale Generators. Recuperado de December 2019, https://www.eia.gov/electricity/monthly/epm table grapher.php?t=epmt 607 b

Unidad de Planeación Minero Energética [UPME]. (2016). Plan de acción indicativo de eficiencia energética. Recuperado

https://www1.upme.gov.co/DemandaEnergetica/MarcoNormatividad/PAI PROURE 2017-2022.pdf

Unidad de Planeación Minero Energética, \& Corpoema-Cusa. (2011). Caracterización Energética del sector residencial urbano y rural en Colombia, Rerporte de medición. Recuperado de http://bdigital.upme.gov.co/jspui/handle/001/1111

US EPA, O. (2018). Greenhouse Gas Equivalencies Calculator. In United States Environmental Protection Agency. Recuperado de https://www.epa.gov/energy/greenhouse-gas-equivalenciescalculator

World Energy Council. (2016). World Energy Resources 2016. Recuperado de https://www.worldenergy.org/wp-content/uploads/2016/10/World-Energy-Resources-Full-report2016.10.03.pdf

XM. (2017). Informe de Operación del SIN y Administración del Mercado 2017. Recuperado de http://informesanuales.xm.com.co/2017/SitePages/operacion/Default.aspx 
Zalamea-León, E.F.; Mena-Campos, J.D.; Moscoso-Cordero, M.S.; Barragán-Escandón, E. A., \& MéndezSantos, P. (2018). Architectural perspectives and photovoltaic roofs in heritage urban contexts. ACE: Architecture, City and Environment, 13(38), 185-210. DOI: https://doi.org/10.5821/ace.13.38.5369

Zandi, M.; Bahrami, M.; Eslami, S.; Gavagsaz-Ghoachani, R.; Payman, A.; Phattanasak, M., ... Pierfederici, S. (2017). Evaluation and comparison of economic policies to increase distributed generation capacity in the Iranian household consumption sector using photovoltaic systems and RETScreen software. Renewable Energy, 107, 215-222. DOI: https://doi.org/10.1016/j.renene.2017.01.051

Zhang, L., \& Wu, Y. (2012). Market segmentation and willingness to pay for green electricity among urban residents in China: The case of Jiangsu Province. Energy Policy, 51, 514-523. DOI: https://doi.org/10.1016/J.ENPOL.2012.08.053

Zubi, G.; Dufo-López, R.; Pardo, N., \& Pasaoglu, G. (2016). Concept development and techno-economic assessment for a solar home system using lithium-ion battery for developing regions to provide electricity for lighting and electronic devices. Energy Conversion and Management, 122, 439-448. DOI: https://doi.org/10.1016/j.enconman.2016.05.075 